$41<79$ mg/L, HR:1.60; $79<137$ mg/L, HR: 2.12; >137 mg/ L, HR: 1.17). The incidence of IMV, ECMO, or death was reduced (OR: 0.31; 95\%CI: $0.15-0.63, \mathrm{p}=0.002$ ) and mortality was improved by 2.22-fold (OR: 2.22; 95\%CI: 1.07-4.67, $\mathrm{p}=0.034)$. In these participants, lenzilumab decreased CRP as early as Day 2 following treatment, compared with placebo which was further decreased by $38 \%$ on Day 28 compared with placebo $(24.4 \pm 3.4 \mathrm{mg} / \mathrm{L}$ vs $39.1 \pm 4.9 \mathrm{mg} / \mathrm{L})$.

Conclusion Lenzilumab significantly improved SWOV in hospitalized, hypoxic participants with COVID-19 pneumonia with the greatest benefits in SWOV and survival in patients with $\mathrm{CRP}<150 \mathrm{mg} / \mathrm{L}$ and age $<85$ years. Inhibition of GM-CSF, an orchestrator of CS, early in the hyperinflammatory response improved outcomes in COVID-19. NCT04351152

\section{S50 A RANDOMISED CLINICAL TRIAL OF AZITHROMYCIN VERSUS STANDARD CARE IN AMBULATORY COVID-19 - THE ATOMIC2 TRIAL}

${ }^{1}$ TSC Hinks, ${ }^{1} \mathrm{~L}$ Cureton, ${ }^{1} \mathrm{R}$ Knight, ${ }^{1} \mathrm{~A}$ Wang, ${ }^{1} \mathrm{JL}$ Cane, ${ }^{1}$ VS Barber, ${ }^{1 J}$ Black, ${ }^{1} \mathrm{SJ}$ Dutton, ${ }^{2} \mathrm{~J}$ Melhorn, ${ }^{1} \mathrm{M}$ Jabeen, ${ }^{3} \mathrm{P}$ Moss, ${ }^{4} \mathrm{R}$ Garlapati, ${ }^{5} \mathrm{~T}$ Baron, ${ }^{6} \mathrm{G}$ Johnson, ${ }^{7} \mathrm{~F}$ Cantle, ${ }^{8} \mathrm{D}$ Clarke, ${ }^{9} \mathrm{~S}$ Elkhodair, ${ }^{10} \mathrm{~J}$ Underwood, ${ }^{11} \mathrm{D}$ Lasserson, ${ }^{1} \mathrm{ID}$ Pavord, ${ }^{1} \mathrm{SB}$ Morgan, ${ }^{1} \mathrm{D}$ Richards. ${ }^{1}$ University of Oxford, Oxford, UK; ${ }^{2}$ University Hospital Llandough, Cardiff, UK; ${ }^{3}$ St George's Hospital, Tooting, London, UK; ${ }^{4}$ East Lancashire NHS Hospitals, Blackburn, UK; ${ }^{5}$ Oxford University Hospitals NHS Foundation Trust, Oxford, UK; ${ }^{6}$ University Hospitals of Derby and Burton, Derby, UK; ${ }^{7}$ Kings College Hospital, London, UK; ${ }^{8}$ Royal Berkshire Hospital, Reading, UK; ${ }^{9}$ University College London Hospital, London, UK; ${ }^{10}$ Cardiff and Vale University Health Board, Cardiff, UK; ${ }^{11}$ City Hospital, Birmingham, UK

\subsection{6/thorax-2021-BTSabstracts.56}

Background The antibacterial, anti-inflammatory and antiviral properties of azithromycin suggest therapeutic potential against COVID-19. Randomised data in mild-moderate disease are lacking. We assessed whether azithromycin is effective in reducing hospitalisation in patients with mild-moderate COVID-19.

Methods This open-label, randomised superiority clinical trial at 19 centres in the United Kingdom enrolled adults, $\geq 18$ years, presenting to hospitals with clinically-diagnosed highlyprobable or confirmed COVID-19 infection, with $<14$ days symptoms, considered suitable for initial ambulatory management. Patients were randomised $(1: 1)$ to azithromycin $(500$ $\mathrm{mg}$ daily orally for 14 days) or to standard care without macrolides. The primary outcome was the difference in proportion of participants with death or hospital admission from any

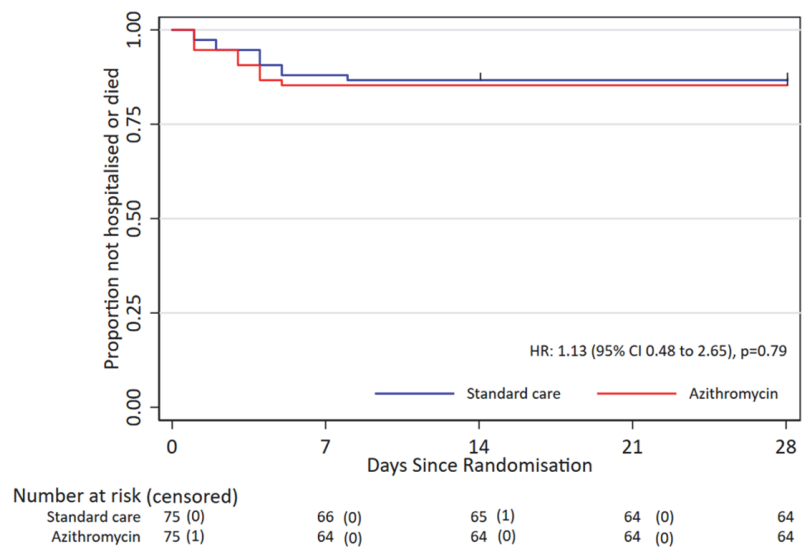

Abstract S50 Figure 1 Kaplan-Meier plot of time to hospitalisation in the ITT +ve population cause over the 28 days from randomisation, assessed according to intention-to-treat (ITT). Trial registration: ClinicalTrials.gov, NCT04381962, Study closed.

Results 298 participants were enrolled from $3^{\text {rd }}$ June 2020 to $29^{\text {th }}$ January 2021. The primary outcome was assessed in 292 participants. The primary endpoint was not significantly different between the azithromycin and control groups (Adjusted OR 0.91 [95\% CI 0.43-1.92], $\mathrm{p}=0 \cdot 80$ ).

Conclusions In patients with mild-moderate COVID-19 managed without hospital admission, adding azithromycin to standard care treatment did not reduce the risk of subsequent hospitalisation or death. Our findings do not support the use of azithromycin in patients with mild-moderate COVID-19.

\section{S51 EVALUATION OF TREATMENT APPROACHES FOR HOSPITALIZED COVID-19 PATIENTS}

A Kilcoyne, E Jordan, C Durrant, D Chappell, O Ahmed. Humanigen, Inc., Burlingame, CA, USA

\subsection{6/thorax-2021-BTSabstracts.57}

Background COVID-19 has driven innovation leading to emergency use authorization of treatments that address its urgent healthcare needs. However, physicians, payers and healthcare systems are challenged to select among these novel treatments for both effectiveness and value. Reliance on change in relative, rather than absolute, risk often makes discrimination of treatment effects between medications impractical, with potentially misleading conclusions. Number Needed to Treat (NNT), the reciprocal of the Absolute Risk Reduction, can be a valuable alternative in assessing benefit:risk. The objective of the current analysis was to calculate NNT for reported endpoints of COVID-19 treatments.

Methods Clinical information was captured from published literature and pre-prints from investigations of COVID-19 treatments. NNTs were calculated for reported endpoints. Outpatient treatments to reduce viral load included neutralizing antibody 'cocktails' REGN-COV2 ${ }^{1}$ and bamlanivimab/etesevimab. ${ }^{2}$ Inpatient treatments included the anti-viral: remdesivir ${ }^{3,4}$; and immune modulators: baricitinib ${ }^{5}$, and lenzilumab. ${ }^{6}$

Results REGN-COV2 reduced the number of medically attended visits with NNT of 33.3. The NNT for hospitalization or death was 20 for bamlanivimab/etesevimab. NNTs for 28-day mortality with inpatient treatment were 37 for baricitinib, 26.3 for remdesivir alone, and 22.7 for lenzilumab. Additional analyses of lenzilumab resulted in NNT of 14.7 when combined with remdesivir and corticosteroids, 15.4 when combined with remdesivir, and 13.9 in patients with baseline $\mathrm{CRP}<150 \mathrm{mg} / \mathrm{L}$ and age $<85$ years. The NNT for lenzilumab to prevent survival without ventilation (SWOV) was 15.4 which, decreased to 6.4 in patients with baseline $\mathrm{CRP}<150 \mathrm{mg} / \mathrm{L}$ and age $<85$ years. The number needed to prevent a serious adverse event was 20 for baricitinib, 15 for remdesivir, and 20.4 for lenzilumab.

Conclusion The NNT for COVID-19 treatments varied with setting, endpoint, and mechanism. The NNT for lenzilumab improved with refinement of concomitant medications and patient phenotype. NNT provides a simple measure for comparative analyses that helps inform clinical decision-making and resource allocation.

\section{REFERENCES}

1. Weinreich, et al. N Engl J Med 2021;384:238-51. 
2. Gottlieb, et al. JAMA 2021;325:632-44.

3. Garibaldi, et al. JAMA NetwOpen 2021:4:e213071.

4. Beigel, et al. N Engl J Med 2020;383:1813-26.

5. Kalil, et al. N Engl J Med 2020.

6. Temesgen, et al. medRxiv 2021.

\section{S52 CONVENTIONAL OXYGEN THERAPY VERSUS CPAP AS A CEILING OF CARE IN WARD-BASED PATIENTS WITH COVID-19: A MULTI-CENTRE COHORT EVALUATION} ${ }^{6} \mathrm{~A}$ Ashish, ${ }^{1} \mathrm{~A}$ Bentley, ${ }^{7} \mathrm{~T}$ Bongers, ${ }^{8} \mathrm{~T}$ Gatheral, ${ }^{1} \mathrm{TW}$ Felton, ${ }^{1} \mathrm{~N}$ Chaudhuri, ${ }^{1} \mathrm{~L}$ Pearmain. ${ }^{1}$ North West Lung centre, Wythenshawe Hospital, Manchester University NHS Foundation Trust, Manchester, UK; ${ }^{2}$ North Manchester General Hospital, Manchester University NHS Foundation Trust, Manchester, UK; ${ }^{3}$ Research and Development, Blackpool Teaching Hospitals NHS Foundation Trust, Blackpool, UK; ${ }^{4}$ NWCORR North West Collaborative Organisation for Respiratory Research study group, North West, UK; ${ }^{5}$ Respiratory Department, Royal Preston Hospital, Lancashire Teaching Hospitals NHS Foundation Trust, Preston, UK; ${ }^{6}$ Respiratory Department, Royal Albert Edward Infirmary, Wrightington, Wigan and Leigh Teaching Hospitals NHS Foundation Trust, Wigan, UK; ${ }^{7}$ Respiratory Department, Blackpool Teaching Hospitals NHS Foundation Trust, Blackpool, UK; ${ }^{8}$ Respiratory department, University Hospitals of Morecambe Bay NHS Foundation Trust, Lancaster, UK

\subsection{6/thorax-2021-BTSabstracts.58}

Background Continuous positive airway pressure (CPAP) therapy is commonly used for respiratory failure due to severe COVID-19 pneumonitis, including in patients deemed unlikely to benefit from invasive mechanical ventilation (nIMV). Little evidence exists demonstrating superiority over conventional oxygen therapy, as acknowledged by current pragmatic guidelines, whilst ward-level delivery of CPAP presents practical challenges. Precedent studies have been limited by small numbers, subjective physician treatment-selection, and lack of a control group. We sought to compare clinical outcomes of oxygen therapy versus CPAP therapy in patients with COVID19 who were nIMV.

Methods The North West Collaborative Organisation for Respiratory Research (NWCORR), a trainee-led network, performed a retrospective multi-centre cohort evaluation. Patient inclusion criteria were: a clinical diagnosis of COVID-19, a treatment escalation plan of ward-level care, treatment at a

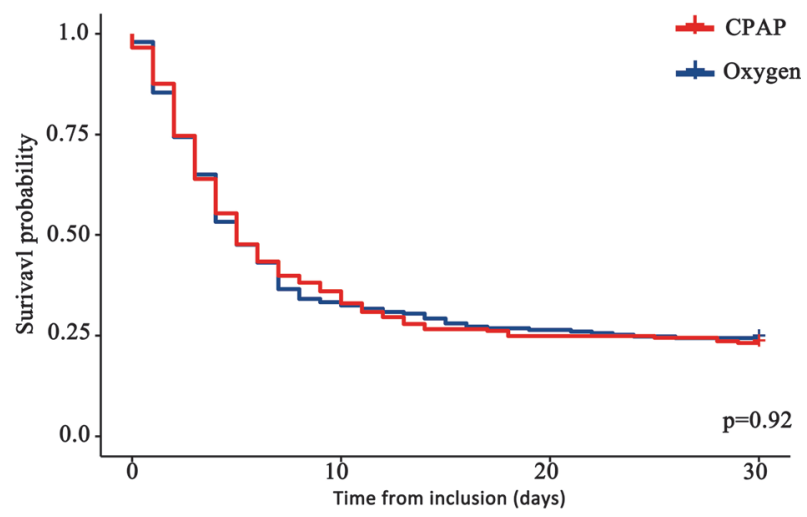

\begin{tabular}{|c|c|c|c|c|}
\hline \multicolumn{5}{|c|}{ Number at risk } \\
\hline Oxygen & 246 & 82 & 65 & 60 \\
\hline CPAP & 233 & 84 & 58 & 54 \\
\hline & 0 & 10 & 20 & 30 \\
\hline
\end{tabular}

Abstract S52 Figure 1 Kaplan-Meier curve comparing overall survival in the two treatment groups (conventional oxygen therapy vs continuous positive airway pressure therapy). The null hypothesis of no survival difference is evaluated with a log-rank test $(p=0.92)$ hospital only providing one respiratory support strategy and clinical frailty score $\leq 6$. Patients were cohorted according to respiratory support strategy, either being CPAP in accordance with national guidelines or oxygen therapy requiring $\mathrm{FiO}_{2}$ $\geq 0.4$ for over 12 hours; who would therefore have been eligible for CPAP if treated at a CPAP cohort hospital. Logistic regression modelling, using generalised estimating equations to account for within-hospital clustering, was performed to compare 30-day mortality between treatment groups, accounting for important confounders.

Results Seven hospitals provided data for 479 patients during the UK COVID-19 pandemic in 2020. Overall 30-day mortality was $75.6 \%$ in the oxygen group (186/246 patients) and $77.7 \%$ in the CPAP group (181/233 patients) (figure 1). A lack of evidence for a treatment effect persisted in the adjusted model (adjusted 30-day mortality odds ratio comparing CPAP to oxygen of $0.84,95 \%$ CI $0.57-1.23, \mathrm{p}=0.37$ ). $49.8 \%$ of patients receiving CPAP-therapy (118/237) chose to discontinue it.

Discussion This is, as far as we are aware, the first study comparing conventional oxygen therapy with CPAP in cohorts unaffected by physician selection. No survival difference was found between using oxygen alone or CPAP to treat patients with severe COVID-19 who were nIMV. A high patient-initiated discontinuation rate for CPAP suggests a significant treatment burden. Further reflection is warranted on the continued widespread use of CPAP in this patient group.

Please refer to page A189 for declarations of interest related to this abstract.

\section{S53 IMPACT OF EMPIRICAL ANTIBIOTIC USE IN PATIENTS WITH COVID-19 ON MORBIDITY AND MORTALITY DURING THE FIRST AND SECOND UK SARS-COV-2 WAVES}

S Waring, G Gamtkitsulashvili, S Kumar, Y Narayan, A D'Souza, S Jiwani, O Taylor, G Collins, K Patrick, A Sethuraman, S Naik, S Kuckreja, R Ragatha, M Anwar, U Ekeowa, P Russell. The Princess Alexandra Hospital, Harlow, Essex, UK

\subsection{6/thorax-2021-BTSabstracts.59}

Background Poor antimicrobial stewardship is frequently observed in COVID-19 patients and relates to greater mortality when empirical antibiotics are administered without compelling evidence of bacterial co-infection. ${ }^{1}$ Here, we assess the impact of antibiotic administration in COVID-19 patients on inpatient morbidity and mortality during the first and second UK SARS-CoV-2 waves.

Methods Two representative four-month timeframes of RTPCR positive COVID-19 admissions to a Greater London District General Hospital were retrospectively analysed, with 481 patients between 15th February 2020 and 15th June 2020 representing the first wave, and 1342 between $1^{\text {st }}$ November 2020 and 28th February 2021 for the second wave. Morbidity was measured by mean length-of-stay. Independent correlation was assessed with multilinear regression analysis adjusting for demographics and comorbidities.

Results $87.9 \%$ of first and $86.0 \%$ of second wave inpatients received at least one antibiotic, despite only $21.4 \%$ and $16.8 \%$ showing bacterial culture positivity of a non-contaminant pathogen, respectively. A mean 2.41 and 2.05 antibiotic types were administered per patient during first and second COVID-19 waves. Antibiotic administration was independently 